\title{
Age, but not short-term intensive swimming, affects chondrocyte turnover in zebrafish vertebral cartilage
}

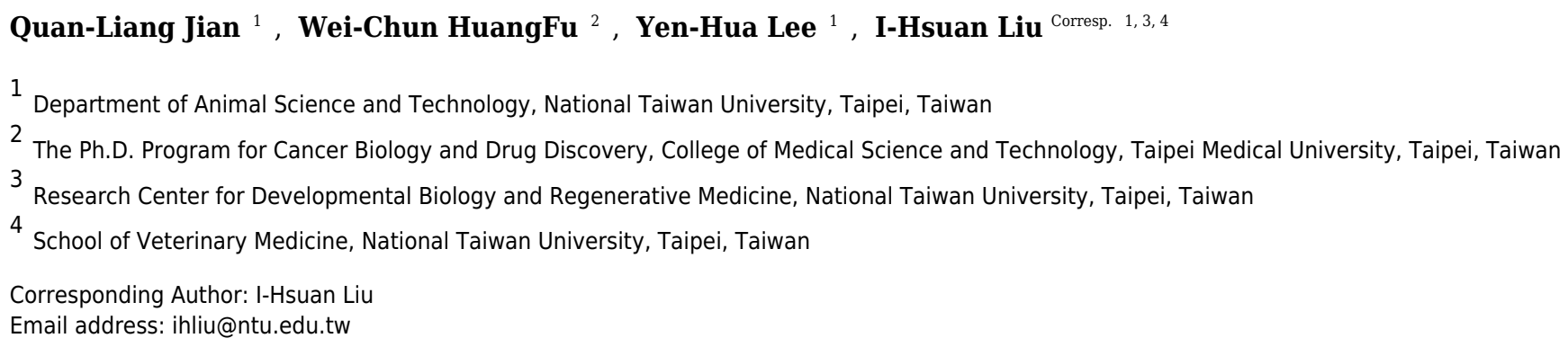

Both age and intensive exercise are generally considered critical risk factors for osteoarthritis. In this work, we intend to establish zebrafish models to assess the role of these two factors on cartilage homeostasis. We designed a swimming device for zebrafish intensive exercise. The body measurements, bone mineral density and the histology of spinal cartilages of 4- and 12-month-old zebrafish, as well the 12-month-old zebrafish before and after a two-week exercise were compared. Our results indicate that both age and exercise affect the body length and body weight, and the microCT reveals that both age and exercise affect the spinal bone mineral density. However, quantitative analysis of immunohistochemistry and histochemistry indicate that short-term intensive exercise does not affect the extracellular matrix (ECM) of spinal cartilage. On the other hand, the cartilage ECM significantly grew from 4 to 12 months of age with an increase in total chondrocytes. TUNEL staining shows that the percentages of apoptotic cells significantly increase as the zebrafish grows, whereas the BrdU labeling shows that proliferative cells dramatically decrease from 4 to 12 months of age. A 30-day chase of BrdU labeling shows some retention of labeling in cells in 4-month-old spinal cartilage but not in cartilage from 12-month-old zebrafish. Taken together, our results suggest that zebrafish chondrocytes are actively turned over, and indicate that aging is a critical factor that alters cartilage homeostasis. Zebrafish vertebral cartilage may serve as a good model to study the maturation and homeostasis of articular cartilage. 
1 Age, but not short-term intensive swimming, affects 2 chondrocyte turnover in zebrafish vertebral cartilage

3 Quan-Liang Jian ${ }^{1}$, Wei-Chun HuangFu², Yen-Hua Lee ${ }^{1}$, I-Hsuan Liu ${ }^{1,3,4}$

$4{ }^{1}$ Department of Animal Science and Technology, National Taiwan University, Taipei

5 106, Taiwan

$6 \quad 2$ The Ph.D. Program for Cancer Biology and Drug Discovery, College of Medical

7 Science and Technology, Taipei Medical University, Taipei, 110, Taiwan

$8{ }^{3}$ Research Center for Developmental Biology and Regenerative Medicine, National

9 Taiwan University, Taipei 106, Taiwan

104 School of Veterinary Medicine, National Taiwan University, National Taiwan

11 University, Taipei 106, Taiwan

12

13 Correspondence author:

14 I-Hsuan Liu

15

16 Email address: ihliu@ntu.edu.tw

17

18

19 


\section{ABSTRACT}

Both age and intensive exercise are generally considered critical risk factors for osteoarthritis. In this work, we intend to establish zebrafish models to assess the role of

23 these two factors on cartilage homeostasis. We designed a swimming device for zebrafish

24 intensive exercise. The body measurements, bone mineral density and the histology of

25 spinal cartilages of 4- and 12-month-old zebrafish, as well the 12-month-old zebrafish before

26 and after a two-week exercise were compared. Our results indicate that both age and

27 exercise affect the body length and body weight, and the microCT reveals that both age and

28 exercise affect the spinal bone mineral density. However, quantitative analysis of

29 immunohistochemistry and histochemistry indicate that short-term intensive exercise does

30 not affect the extracellular matrix (ECM) of spinal cartilage. On the other hand, the cartilage

31 ECM significantly grew from 4 to 12 months of age with an increase in total chondrocytes.

32 TUNEL staining shows that the percentages of apoptotic cells significantly increase as the

33 zebrafish grows, whereas the BrdU labeling shows that proliferative cells dramatically

34 decrease from 4 to 12 months of age. A 30-day chase of BrdU labeling shows some

35 retention of labeling in cells in 4-month-old spinal cartilage but not in cartilage from 12-

36 month-old zebrafish. Taken together, our results suggest that zebrafish chondrocytes are

37 actively turned over, and indicate that aging is a critical factor that alters cartilage

38 homeostasis. Zebrafish vertebral cartilage may serve as a good model to study the

39 maturation and homeostasis of articular cartilage. 


\section{Manuscript to be reviewed}

\section{INTRODUCTION}

42 Osteoarthritis (OA) is the most common pathologic condition of articular cartilage

43 and leads to joint pain and stiffness, degeneration of articular cartilage and, 44 sometimes, ectopic osteogenesis to form osteophytes. It is generally believed that 45 both age and mechanical loading are critical risk factors for OA. Accordingly, 46 previous reports indicate that age is positively correlated to the prevalence of OA 47 and elite athletes have a higher risk of OA and arthroplasty (Busija et al. 2010; Tveit 48 et al. 2012). More importantly, articular cartilage poorly regenerates, and OA is 49 generally considered only treatable, but incurable.

50 Histologically, cartilage can be categorized into three major types: hyaline 51 cartilage, fibrocartilage and elastic cartilage. Articular cartilage is hyaline cartilage, which predominantly contains a homogenous and translucent extracellular matrix $(E C M)$ and is covered by perichondrium. The ECM in the articular cartilage is mostly type II collagen with some other collagens, proteoglycans and glycosaminoglycans (GAGs) such as hyaluronan, chondroitin sulfate and keratan sulfate. The cells only account for a small portion of the volume in articular cartilage. In mammalian joints, the articular cartilage can be divided into four regions including a superficial zone, middle zone, deep zone and calcified zone. The superficial zone contains the highest cell density and the superficial cells secret proteoglycan 4 as a joint lubricant. These cells have a large long/short morphological axis ratio (Schumacher et al. 1994). The middle zone accounts for the major volume of an articular cartilage and the middle cells are enlarged, with an oval shape, usually sitting in lacunae compared to the superficial cells (Hedlund et al. 1999). The deep cells are usually round hypertrophic chondrocytes, while the calcified zone is a transition region between cartilage and subchondral bone (Grogan et al. 2009; Schmid \& 66 Linsenmayer 1985).

Due to its avascular and aneural nature, articular cartilage was historically believed to be inert. Later, injection of radioactive isotopes indicates a dynamic change in the composition of GAGs and indicates that cartilage is not completely lack of metabolism (Davidson \& Small 1963; Mankin \& Lippiello 1969). Studies taking advantage of the fluctuation of atmospheric ${ }^{14} \mathrm{C}$ support the notion that GAGs are dynamically turned over, but also reveal that collagen in the articular cartilage is extremely inert (Heinemeier et al. 2016; Libby et al. 1964). Moreover, studies in the recent decade suggest that superficial cells might work as stem cells that supply new chondroblasts for cellular turnover in the articular cartilage (Alsalameh et al. 2004; Candela et al. 2014; Dowthwaite et al. 2004). It is now widely believed that cellular turnover supported by endogenous stem cells occurs in the articular cartilage during 78 young ages but not in mature articular cartilage, which might result in the age-related 79 risk for OA.

Since buffering mechanical loading is one of the physiological functions of 81 articular cartilage, it is reasonable to expect articular cartilage to bear a certain 82 mechanical load. Joint cartilages that are immobilized for weeks to months result in 
83 signs of OA including loss of GAGs and formation of osteophytes (Jurvelin et al.

84 1985; Langenskiold et al. 1979; Videman et al. 1981). On the other hand, not only 85 professional athletes have a higher prevalence of OA, but articular cartilage in 86 animals that receive rigorous exercise training also show OA-like changes (Arokoski 87 et al. 1993; Kujala et al. 1994; Lequesne et al. 1997; Paukkonen et al. 1985; 88 Saamamen et al. 1994; Tveit et al. 2012). Although the mechanisms for mechanical 89 loading to affect articular cartilage remains elusive, multiple lines of evidence 90 suggest that a moderate level of mechanical loading is beneficial to the articular 91 cartilage (Kiviranta et al. 1988; Saamanen et al. 1990).

92 Zebrafish have emerged as an excellent model to study embryonic development 93 as well as tissue regeneration, but they could also serve as a model to study 94 homeostasis and aging. Previous study indicates that ability and trainability of 95 physical exercise declines with the age of zebrafish, similar to mammals (Gilbert et 96 al. 2014). Furthermore, not only is vertebral cartilage development and maturation 97 promoted by exercise training, aging also leads to deformity of vertebral cartilage 98 that recapitulates signs of OA (Fiaz et al. 2012; Hayes et al. 2013). In this study, we 99 100 101

102 aimed to determine whether age and exercise training affect the homeostasis of vertebral cartilage in adult zebrafish by evaluating the content of GAGs and type II collagen as well as cellular dynamics.

\section{MATERIALS AND METHODS}

\section{Zebrafish strain and maintenance}

104

105

106

107

108

109

110

111

112

113

114

The $A B$ wild-type line of zebrafish purchased for exercise experiments (GenDanio Aquaculture system, New Taipei City, TW) were randomly segregated into two groups: control (Ctrl) vs. exercise (Exe), and all the comparisons between before $(+0 d)$ vs. after $(+14 d)$ exercise training program were done with these zebrafish. The $A B$ wild-type line of zebrafish at 3 to 4 months of age and 11 to 13 months of age were purchased (Azoo Co., Taipei, TW) for the age comparisons (4vs. 12 -month-old). All zebrafish were kept individually (in $200-\mathrm{mL}$ water) at $28.5^{\circ} \mathrm{C}$ with a light cycle of 14-hour light/10-hour dark and were fed twice daily for this study. All experimental procedures in this study were reviewed and approved by the Institutional Animal Care and Use Committee (IACUC) of National Taiwan University (NTU105-EL-00037) and were performed in accordance with the approved guidelines.

\section{Zebrafish intensive exercise training}

To force zebrafish to go through intensive exercise training, a simple training system was designed and assembled with an aquatic powerhead (Rio+1400,

120 Technological Aquatic Association Manufacturing, Thousand Oaks, CA) connected 121 to a polyisoprene tube ( $52 \mathrm{~cm}$ in length, $2.5 \mathrm{~cm}$ in diameter) and a mesh covering the 
122 end opening (Figure 1A). Each system housed an individual zebrafish in the tube

123 during the training session and a 60-liter polyethylene tank housed 3 training

124 systems placed in a stack fashion.

125 Each zebrafish in the exercise group was assessed for their maximal resisting 126 speed. Briefly, the flow of the aqua pump was increased every minute until the 127 zebrafish fail to resist and reside the mesh (fail speed). The maximal speed (i.e., the 128 flow speed immediately before the fail speed) was then calculated according to the 129 milli-liter-per-minute of the aqua pump and the cross-sectional area of the tube 130 (Table 1). Each zebrafish in the exercise group was transferred into the system 30 131 min after the morning feed and rested for 30 min before the 8-hour training session 132 at maximal speed began (Figure 1B). After the training session, the zebrafish was 133 transferred back to the housing system, received an excessive night feed. The 134 training session lasted for 14 days, and the control group in this experiment was 135 managed in the same way without turning the aqua pump on.

\section{Body measurements}

137 To assess the effects of age and exercise training on overall physiological 138 condition, body weight and body length of each zebrafish were measured and 139 recorded. Briefly, each zebrafish was anesthetized in $0.016 \%$ ethyl 3140 aminobenzoate methanesulfonate (MS-222, Sigma-Aldrich, St. Louis, MO, USA) 141 before the morning feed. The body weight was measured on a precision balance 142 (PJ3600, Mettler-Toledo, Columbus, $\mathrm{OH}$ ) after excessive water was removed and a 143 photograph was taken to measure the body length from the mouth tip to the end of 144 the tail-fin using ImageJ (Schneider et al. 2012).

\section{Micro-computed tomography and bone mineral density}

To assess the effects of age and exercise training on the skeletal system, bone mineral density (BMD) of each zebrafish was estimated using images from microcomputed tomography (microCT). Briefly, zebrafish were anesthetized using a mixture of $100 \mathrm{ppm} \mathrm{MS-222}$ and 100 ppm isoflurane (Abbott Laboratories, Queenborough, UK) in water (Huang et al. 2010), restrained between two wet sponges, and scanned (SkyScan-1076, Bruker microCT, Kontich, BE) with $9 \mu \mathrm{m}$ resolution, $80 \mathrm{kV}, 124 \mu \mathrm{A}, 0.5^{\circ}$ rotational step, $1700 \mathrm{~ms}$ exposure and a $0.5 \mathrm{~mm}$ aluminium filter (Table S1). To standardize and calibrate the intensity, two scanning phantoms with 0.25 and $0.75 \mathrm{~g} / \mathrm{cm}^{3}$ of densities were used. The scale was designed according to a pre-defined parameter (air, $\mathrm{HU}=-1000$, color index=0) and scanning results (including water and phantoms) to generate the mapping reference between color index (0 to 255) and Housefield units (HU; in our case, -1000 to 3184). The 3D rendering was done by using CTvox software (Bruker microCT) and the BMD was analyzed by using CTAn software (Bruker microCT) with the fourth (a Weberian vertebra) or all vertebrae as the region of interest (Bird \& Mabee 2003; Hur 2017). 
162

163

164

165

166

167

168

169

170

171

172

173

174

175

176

177

178

179

180

181

182

183

184

185

186

187

188

189

190

191

192

193

194

195

196

197

198

To understand the effect of age and short-term intensive exercise training on chondrocyte proliferation, 5-bromo-2'-deoxyuridine (BrdU; Sigma-Aldrich) was used to label the proliferative cells. For age comparisons, the zebrafish were anesthetized in $0.016 \% \mathrm{MS}-2223 \mathrm{~h}$ after the night feed with $5 \mu \mathrm{L}$ of BrdU $(2.5 \mathrm{mg} / \mathrm{mL}$ in distilled water) were administered via oral gavage once a day for consecutive $15 \mathrm{~d}$ (Reimer et al. 2008). To reduce stress for the exercise comparisons, zebrafish were immersed in $200 \mathrm{~mL}$ of $\mathrm{BrdU}(150 \mu \mathrm{g} / \mathrm{mL})$ each day during the dark period of the 2week training session (Rowlerson et al. 1997). To determine whether quiescent cells exist in the cartilage, the BrdU labeled zebrafish were chased for an additional $30 \mathrm{~d}$.

\section{Histology preparation}

To observe the effect of age and short-term intensive exercise training on the morphology and composition of cartilage, qualitative and quantitative histology were analyzed. Briefly, the zebrafish were sacrificed in $20 \mathrm{~mL} 0.4 \% \mathrm{MS}-222$, and fixed in $20 \mathrm{~mL} 4 \%$ paraformaldehyde (Merck, Darmstadt, DE) at $4{ }^{\circ} \mathrm{C}$ for $5 \mathrm{~d}$ after the abdomen was opened with a scalpel for better penetration of the fixative. The zebrafish were then immersed in $20 \mathrm{~mL}$ of $10 \%$ ethylenediaminetetraacetic acid (EDTA, Amresco, Solon, OH, USA) for $3 \mathrm{~d}$ for de-calcification. After the fixatives and EDTA were washed away with water, dehydration was with $20 \mathrm{~mL}$ each of a 30 $100 \%$ ethanol gradient. The sample was then immersed two times in $20 \mathrm{~mL}$ of xylene (J.T. Baker, Center Valley, PA, USA) for $1 \mathrm{~h}$ each and embedded in paraffin (Surgipath Medical Industries, Richmond, IL). The tissue blocks were sectioned at 5 $\mu \mathrm{m}$ to produce consecutive sagittal sections using a rotary microtome (HM315, Microm, Walldorf, DE). The tissue slides were then rehydrated using xylene followed by a $100-30 \%$ ethanol gradient, and finally immersed two times in phosphate buffered saline (PBS; Amresco) for $5 \mathrm{~min}$. From the most lateral edge of the vertebral column to the midline, about 30 (in 4-month-old) or 40 (in 12-month-old) consecutive sections could be obtained. For all quantitative image analysis, 5 sections from the same subject with consistent $20 \mu \mathrm{m}$ (in 4-month-old) or $25 \mu \mathrm{m}$ (in 12-month-old) interval between sections were used for the same staining analysis and the sum of the results from 5 slides represented the result for the subject.

\section{Histochemistry}

To observe the GAG content in the cartilage, the tissue slides were immersed in hematoxylin (Surgipath Medical Industries) for $5 \mathrm{~min}$, washed with $1 \% \mathrm{HCl}$ (Merck) and distilled water, immersed in $0.02 \%$ fast green (Merck) for $1 \mathrm{~min}$, washed with $1 \%$ acetate (Merck) and distilled water, immersed in safranin $O$ (Merck) for $10 \mathrm{~min}$, washed with $95 \%$ and $100 \%$ ethanol, and finally sealed with mounting medium (Muto Pure Chemicals, Tokyo, JP). 


\section{Immunohistochemistry}

200 To observe the distribution of type II collagen and BrdU labeling/retention in the 201 cartilage, immunohistochemistry was performed. Briefly, after the tissue slides were 202 rehydrated, epitope retrieval was achieved by proteinase K $(20 \mu \mathrm{g} / \mathrm{mL}$; GeneMark, 203 Taichung, TW) incubation at room temperature for $30 \mathrm{~min}$ (for type II collagen). For $204 \mathrm{BrdU}$, incubation was with sodium citrate $2.94 \mathrm{mg} / \mathrm{mL}, \mathrm{pH} 6.5$; Sigma-Aldrich) at 205 step-up temperatures from 65 to $95{ }^{\circ} \mathrm{C}$ for $10 \mathrm{~min}$. After washing with $0.1 \%$ Tween20620 (Amresco) in PBS (PBST) twice, the tissue slides were blocked with blocking 207 buffer (3\% bovine serum albumin (Sigma-Aldrich) in PBS) at room temperature for $20830 \mathrm{~min}$ and incubated with the primary antibodies against type II collagen (1:10 in 209 blocking buffer; Developmental Studies Hybridoma Bank, lowa City, IA) for $2.5 \mathrm{~h}$ or 210 against BrdU (1:100 in blocking buffer; AbD Serotec, Kidlington, UK) for $30 \mathrm{~min}$ at 211 room temperature. After washing with $0.1 \%$ Tween-20 in PBS, the primary 212 antibodies were detected by goat anti-mouse IgG conjugated with Alex Fluor 555 213 (1:300 in blocking buffer; Abcam, Cambridge, UK) for $1 \mathrm{~h}$ at room temperature. The 214 nuclear counter-staining was done by 4',6-diamidino-2-phenylindole (DAPI, 10 $215 \mathrm{mg} / \mathrm{mL}$; Biotium, Fremont, CA, USA) and the tissue slides were sealed with 216 Fluoroshield mounting medium (Abcam). A primary-free, secondary-only antibody 217 staining was used as a negative control.

\section{Terminal deoxynucleotidyl transferase dUTP nick end labeling 219 (TUNEL)}

To assess the effect of age and exercise training on chondrocyte apoptosis, the terminal deoxynucleotidyl transferase dUTP nick end labeling (TUNEL) assay was performed using a commercial kit (In Situ Cell Death Detection Kit, TMR red; Roche, Basel, $\mathrm{CH}$ ). Briefly, after rehydration, the tissue slides were perforated with proteinase $\mathrm{K}(20 \mu \mathrm{g} / \mathrm{mL})$ for $15 \mathrm{~min}$ at room temperature and washed with PBST. The tissue slides were then incubated with the terminal deoxynucleotidyl transferase (TdT) and dUTP mixture $(1: 10)$ at $37{ }^{\circ} \mathrm{C}$ for $1 \mathrm{~h}$, counter stained with DAPI, and sealed. A DNase I (2 U/ML; Geneaid Biotech, Taipei, TW) treatment at room temperature for $10 \mathrm{~min}$ after perforation was used as a positive control, while a TdTfree reaction was used as a negative control for this staining.

\section{Micrographs and image analysis}

All the tissue slides were documented by a tissue scanner (TissueFAXS, TissueGnostics, Vienna, AT) or by a confocal microscope equipped with differential interference contrast (DIC) (TCS SP5 II, Leica Microsystems, Wetzlar, DE). For the quantitative image analysis, 3 (for type II collagen) or 5 slides from the same subject with a consistent interval between sections were used for the same staining analysis.

236 The sum of the results represented the subject. The software HistoQuest 237 (TissueGnostics) was used to quantitatively analyze the contents of GAGs and type 
238 II collagen in the cartilage, while ImageJ was used to count the nuclei with DAPI,

239 BrdU or TUNEL staining (Schneider et al. 2012).

\section{Scanning electron microscope (SEM)}

241 For the SEM imaging, the tissue sections were collected onto the cover slips (32

$242 \times 24 \mathrm{~mm}$ ). The sections were then de-waxed twice in xylene for $10 \mathrm{~min}$, then three

243 times in 100\% ethanol for 10 min each followed by two immersions in acetone for 10

244 min. Each slide was then critical point dried in liquid $\mathrm{CO}_{2}$ in a critical point dryer

245 (Hitachi, Tokyo, JP) and ion coated (IB-2, Eiko, Tokyo, JP) before documentation in

246 the scanning electron microscope (Inspect S, FEI, Hillsboro, Oregon, USA).

\section{Statistical analysis}

248 To minimize the potential bias brought about by small sample sizes, non249 parametric statistical approaches were used in this study. To minimize the influence 250 due to individual variations, the Wilcoxon matched-pairs signed rank test was used 251 for the comparisons between before and after exercise training sessions. For the 252 comparisons between age groups (4-month-old vs. 12-month-old) and exercise 253 groups (exercise vs. control), the Mann-Whitney U-test was used. For the 254 comparisons among 0-, 15- and 30-day chase, Kruskal-Wallis test was used. 255 Statistical significance was considered when $\mathrm{P} \leq 0.05$.

\section{RESULTS}

\section{Zebrafish continues to grow after sexual maturity while intensive 258 exercise hinders this growth}

259

260

261

262

263

264

265

266

267

268

269

270

271

272

273

In mammals, hormones such as estrogen fluctuate dramatically during sexual maturity and trigger the halt in skeletal growth including the closure of epiphyseal plates in bones (Zhong et al. 2011). The body measurements of zebrafish indicate that zebrafish continues to grow after sexual maturity as the body length increased significantly from $2.65 \mathrm{~cm}$ at 4 months of age to $3.12 \mathrm{~cm}$ (Figure 1C, Table S2). Intriguingly, although the body length of the control zebrafish was not significantly changed in 2 weeks, the body length of zebrafish was significantly shortened after the two-week intensive exercise-training program (Figure 1D, Figure S1, Table S2).

Similarly, the body weight was significantly increased from $0.16 \mathrm{~g}$ in 4-month-old zebrafish to $0.23 \mathrm{~g}$ at 12 months of age (Figure 1E, Table S3). The body weight continued to increase during the 2-week experimental period in the 12-month-old control group, but the body weight was not altered in the zebrafish experiencing intensive exercise training (Figure 1F, Table S3). These results indicate that the zebrafish body continues to grow between 4 and 12 months of age, especially the body weight, whereas short-term intensive exercise halts the growth. 
274 The BMD continues to increase after sexual maturity while

275 intensive exercise negatively affects this trend

276 Previous studies in the human skeletal system indicate that the BMD peaks 277 between 30 to 40 years of age. Appropriate nutrition and exercise enhance the BMD 278 level, but aging especially in females after 50 years of age increases the risk of 279 osteoporosis and OA (Lee et al. 2013; Warming et al. 2002). To assess the potential 280 impact of short-term intensive exercise on the vertebrae column, microCT scan with 281 the fourth vertebrae selected as region of interested was used to estimate the BMD 282 (Figure 2A, B). The result shows that the BMD in the fourth vertebrae continued to 283 grow significantly in the control group during the 2-week experimental period (Figure 284 2C, Table S4). The BMD level remained at comparable levels before and after the 285 2-week intensive exercise training in the exercise group (Figure 2D, Table S4). 286 These results indicate that zebrafish BMD level in the fourth vertebrae continues to 287 increase even at 12 months of age, but the short-term intensive exercise hinders this 288 increase in BMD.

\section{9 \\ 290 \\ Zebrafish continue to accumulate cartilage ECM after sexual maturity}

291

292

The GAGs and collagens, especially type II collagen, are the predominant

293

294

295

296

297

298

299

300

301

302

303

304

305 constituents of articular cartilage, and the networking of these macromolecules buffers and disperses the mechanical pressures applied to the joints (Hedlund et al. 1999; van der Rest \& Mayne 1988). One of the signatures of OA is the loss of these ECM components (Pritzker et al. 2006). Previous study shows that, as the zebrafish ages, the fourth and fifth vertebrae develop bone and cartilage deformities similar to OA symptoms in humans (Hayes et al. 2013). To assess the effects of age and mechanical loading on ECM accumulation in cartilage, histochemistry, immunohistochemistry and the quantitative analysis of micrographs were performed with the fourth Weberian vertebra (Figure $3 A$ and $B$ ) as the region of interest since it includes the largest cartilage ECM, including type II collagen (Figure $3 \mathrm{~A}$ ) and GAGs (Figure 3B), compared to other vertebrae. Furthermore, the cartilage at the fourth Weberian vertebra resembles the histological features of hyaline cartilage as it contains no prominent collagen fibers (bracket in Figure $3 \mathrm{C}$ ) and is surrounded by fibrous perichondrial organization (arrow and bracket in Figure 3D).

306

307

Interestingly, the distribution of type II collagen was more prominent at the ventral and dorsal end of the cartilage in 4-month-old zebrafish (Figure 4A), but

308

309 much more prominent at cartilage margins in 12-month-old zebrafish with no discernible difference between exercise and control groups (Figure 4B and C). Quantitative analysis of immunohistochemical micrographs of type II collagen show significant lower levels in both distribution areas (Figure 4D, Table S5) and signal density (Figure 4E, Table S6) in 4-month-old zebrafish compared to 12-month-old zebrafish. On the other hand, both the distribution area (Figure 4F, Table S5) and 
314 signal density (Figure 4G, Table S6) of type II collagen were at comparable levels in

315 exercise and control groups.

316 The histochemistry of safranin $\mathrm{O}$, fast green and hematoxylin can provide well317 discerned depictions of GAGs, collagens and cell nuclei in a tissue (Figure 5A-C).

318 Compared to type II collagen (Figure 4A-C), the GAGs were more prominently 319 distributed in the core of the cartilage, especially in 12-month-old zebrafish, while 320 occupying a larger area in the vertebra (Figure 5A-C). Interestingly, although the 321 GAG area size was significantly smaller in 4-month-old zebrafish than in 12-month322 old zebrafish (Figure 5D, Table S7), the signal densities were at a comparable level 323 (Figure 5E, Table S8). Similar to type II collagen, both the distribution areas (Figure $3245 \mathrm{D}$, Table S7) and signal densities (Figure 5E, Table S8) of GAGs were at 325 comparable levels between exercise and control groups. Taken together, the short326 term intensive exercise training does not result in discernible change in the zebrafish 327 cartilage, while the cartilage continues to grow after the sexual maturity of zebrafish 328 at 4 months of age. Interestingly, the accumulation of type II collagen seems less 329 mature than GAGs in 4-month-old zebrafish, since the signal density of GAGs but 330 not type II collagen remained at comparable levels between 4 and 12 months of age.

\section{Cellular dynamics decreased with age}

332 Since the homeostasis of cartilage ECM, especially the GAGs, depend on the 333 balance of catabolism and anabolism of chondrocytes, it is essential to evaluate the 334 chondrocytes in the cartilage. As hematoxylin staining and the existence of lacunae 335 clearly depicted the distribution of chondrocytes and their nuclei (Figure 5A-C), the 336 cell counts and cell densities were also evaluated using the same tissue slides. The 337 zebrafish vertebral chondrocytes did not distribute with apparent orientations, but the 338 cells lacking surrounding lacunae were predominately located at marginal regions 339 (Figure 5A-C). The 4-month-old zebrafish had significantly greater cell density than 340 the 12-month-old zebrafish (Figure 5F, Table S9), but the cartilage in 12-month-old 341 zebrafish contained more cells (Figure 5G, Table S9). Again, both the cell densities 342 and cell counts were at comparable levels between the exercise and control groups. 343 These results indicate that, between 4 and 12 months of age, the continuous growth 344 of cartilage is contributed both by the accumulation of ECM and by the increase in 345 chondrocytes.

346 To further elucidate whether the increase in chondrocytes was a static 347 accumulation or a result of a dynamic equilibrium, TUNEL staining and BrdU labeling 348 were performed. The TUNEL staining indicates that the apoptotic cells were 349 predominantly located at the outer regions of the vertebral cartilage (most 350 anterior/posterior and dorsal/ventral tips) (Figure 6A, Table S10). Quantitative 351 analysis shows that cartilage in 12-month-old zebrafish contained a significantly 352 greater percentage of apoptotic cells than cartilage in 4-month-old zebrafish (Figure $3536 \mathrm{~B}$ ), whereas the exercise group was not significantly different from the control group 354 (Figure 6B, Table S10). On the other hand, BrdU labeling (Figure 6C, Table S11) 355 indicates that cartilage (within GAG-positive as well as type II collagen-positive 
356 regions) in 4-month-old zebrafish contains far greater percentages of proliferating

357 cells than cartilage in 12-month-old zebrafish, and intensive exercise training does 358 not alter the proliferative potential of chondrocytes (Figure 6D, Table S11). 359 Interestingly, the average percentage of BrdU-positive cells in 12-month-old 360 zebrafish was merely $0.117 \%$ (12-month-old), $0.055 \%$ (control group) and $0.045 \%$ 361 (exercise group) in contrast to the $3.24 \%$ in 4-month-old zebrafish, while there were 362 no BrdU-positive cells in many of the 12-month-old cartilage. These results indicate 363 that the cellular renewal is gradually lost as zebrafish aged.

Although the BrdU-positive cells were sporadically distributed in the cartilage with no specific localizations, some of the BrdU-positive cells were located in the

366

367 peripheral regions where the GAGs and type II collagen were not accumulated (Figure 6C, Table S11). These cells resided at a location resembling perichondrium 368 with elongated nuclei similar to superficial cells in mammalian articular cartilage. Recent studies imply that these perichondrial superficial cells could serve as stem cells or progenitor cells to provide new cells for chondrocyte turnover (Candela et al. 2014; Karlsson et al. 2009; Li et al. 2017). To assess whether stem cell-like

372 quiescent cells are present in zebrafish cartilage, BrdU pulse-chase was performed 373 in attempting to look for cells retaining the label (Figure 6E, Table S12). However, 374 we found no BrdU-positive cells in the entire fourth Weberian vertebra in any 12month-old zebrafish samples (data not shown). In the 4-month-old zebrafish 376 cartilage, the BrdU-positive percentages decreased from $3.24 \%$ to $0.58 \%$ after a 15 day chase and $1.69 \%$ after a 30 -day chase (Figure 6E, Table S12) with no statistical 378

379 significance. Furthermore, as HMGB2 was previously reported a potential molecular marker for mesenchymal stem cell-like chondrocytes in mouse articular cartilage 380 (Taniguchi et al. 2009), immunohistochemistry using anti-HMGB2 antibody also 381 found no cells being labeled in the entire fourth Weberian vertebra in both 4- and 12382 month-old zebrafish (data not shown). Taken together, age indeed affects 383 chondrocyte dynamics and we found no evidence to suggest the existence of cartilage stem cells in mature zebrafish at 12 months of age.

\section{DISCUSSION}

386 In this study, 4- and 12-month-old zebrafish was used to study the effect of age on cartilage homeostasis, especially chondrocyte dynamics. Every zebrafish used in

388 this study demonstrated courtship behavior with female zebrafish and the embryos 389 laid by the female were fertilized indicating the zebrafish, even the 4-month-old ones, 390 were sexually mature. In humans and other mammals, "body maturity" usually 391 comes after "sexual maturity". Although different body parts vary dramatically, it is 392 generally accepted that the human body reaches full maturity between 20 to 30 393 years of age and then remains static between 20 to 50 years of age. In our results, it 394 was apparent that the zebrafish continued to grow even after sexual maturity (Figure 395 1C, E), and therefore body maturity comes after sexual maturity in zebrafish. In line 396 with our result, a previous study indicates that, after sexual maturity, zebrafish 
397 continue to grow at least up to 9 months of age (Parichy et al. 2009). The vertebral 398 BMD of zebrafish also showed a significant increase in the control group during the 399 2-week exercise study period (Figure 2C). A previous study shows that, although 400 morphologically changed, the BMDs of the 5th vertebrae of zebrafish are developed 401 at a comparable level at 12, 24 and 36 months of age (Hayes et al. 2013). Taken 402 together, it is likely that the zebrafish reaches full body maturity between 9 to 24 403 months of age, and probably begins to show signs of aging after 24 months of age 404 without significantly losing BMD.

405 To evaluate the effect of mechanical loading on cartilage homeostasis in mature 406 zebrafish, a simple intensive-exercise-training system was designed and assembled 407 (Figure 1A). The maximal swimming speed of $22.4 \mathrm{~cm} / \mathrm{s}$ is very similar to our test 408 result and indicates that our system could provide intensive exercise training to 409 zebrafish (Gilbert et al. 2014). Our results showed different changes after a 14-day 410 period of intensive exercise training compared to the control group (Figure 1D and 411 F). Among these changes, a significantly shorter body length after a 2-week training 412 program (Figure 1D) is most surprising and intriguing to us. We attempted to 413 determine the curvatures of the spines using the microCT dataset with no apparent 414 correlative changes (Table S13). One of the tempting speculations to explain this 415 result is the different growth and tone of the musculatures between two groups, as a 416 previous study shows that exercise ability of zebrafish is still trainable at this age 417 (Gilbert et al. 2014).

418 It is widely accepted that exercise is beneficial to BMD accumulation and can 419 ameliorate the loss of BMD (Shimegi et al. 1994). Furthermore, exercise training in zebrafish larvae stimulates the progress of early endochondral ossification including the Weberian vertebrae, suggesting that the development of the skeletal system 422 indeed is affected by increased mechanical loading (Fiaz et al. 2012). However, we 423 found that 2-week intensive exercise training negatively affected the BMD 424 accumulation (Figure 2C and D). Interestingly, previous studies indicate that, 425 although some sports positively affect BMD in specific bones, swimming does not 426 positively affect BMD (Bennell et al. 1997; Ferry et al. 2013; Magkos et al. 2007; 427 Maimoun et al. 2013). It is possible that, although the dynamic homeostasis of the 428 skeletal system is affected by mechanical loading, gravity contributes a critical role in 429 this mechanical loading, while buoyancy provided by water minimizes the effect of 430 gravity and hence the BMD of zebrafish is predominantly affected by age and 431 perhaps energy balance (Siccardi et al. 2010). In our study, every zebrafish was 432 individually housed and fed with excessive amounts of food, and therefore the 433 possibility that nutritional insufficiency due to housing or dietary intake could be 434 minimized. In contrast, previous studies suggest that increased exercise in zebrafish 435 promotes catabolic genes such as citrate synthase or nuclear respiratory factor 436 (NRF-1) (Liu \& Wang 2013; McClelland et al. 2006). Therefore, we speculate that 437 our intensive exercise training caused a surge in catabolism and in turn hindered the 438 accumulation of BMD and general body mass. Although the BMD in the fourth 439 vertebrae was indeed affected by the exercise training, none of our results showed 
440 any difference of cartilages between exercise and control groups. Considering that

441 anterior one-third of the body stays rigid during an adult zebrafish swimming 442 (Fontaine et al. 2008; Muller et al. 2000), the pre-caudal vertebrae, including the 443 fourth Weberian vertebrae, are probably not bearing the mechanical load in a similar 444 way as a mammalian articular cartilage during exercise. Therefore, despite that the 445 cartilage in this area is affected by exercise in zebrafish larvae (Fiaz et al. 2012), it is 446 possible that this model did not provide sufficient mechanical load to cartilage and 447 zebrafish vertebral cartilage was not affected by swimming in 12-month-old 448 zebrafish.

449 In our observations, type II collagen was more prominently stained in the 450 cartilage margins (Figure 4A-C), while GAGs were more prominently stained in the 451 cartilage core (Figure 5A-C). To our knowledge, this inconsistency was not 452 described in other articular cartilage, and the generally accepted notion suggests 453 that type II collagen, proteoglycans and GAGs intermingle to constitute the ECM of 454 articular cartilage (van der Rest \& Mayne 1988). Furthermore, current evidence suggests that collagen fibers in human articular cartilage mature during teenage years with extremely limited turnover and increase after the age of 20 (Heinemeier et al. 2016; Libby et al. 1964). Our results indicate that both the occupying area and 458 the signal density for type II collagen were increased from 4 to 12 months of age 459 (Figure 4D and E). This result supported our previous speculation that the body 460 maturity of zebrafish came between 9 and 12 months of age. On the other hand, 461 while the collagens in the articular cartilage are extremely inert, the GAGs are 462 dynamically metabolized (Heinemeier et al. 2016; Libby et al. 1964; Mankin \& 463 Lippiello 1969). Accordingly, our result indicates that the signal density for GAGs 464 was already saturated in 4-month-old zebrafish (Figure 5E). During the increase in 465 occupying area (Figure 5D), the total amount of GAGs might increase in a linear 466 fashion. Previous study indicates that chondroitin sulfate, the predominant type of 467 GAG in articular cartilage, increases in a linear fashion as zebrafish age from 1 to 3 468 years (Hayes et al. 2013).

469 The loss of chondrocytes has been considered one of the reasons for the age470 related degeneration of cartilage (Barbero et al. 2004; Stockwell 1967). In the 471 vertebral cartilage, 2- and 3-year-old zebrafish contain more total lacuna area than 1472 year-old zebrafish (Hayes et al. 2013). Two possible explanations could be 473 deduced: (1) old zebrafish have more hypertrophic chondrocytes or (2) old zebrafish 474 lost more chondrocytes. In this study, we attempted to perform 475 immunohistochemistry against type $X$ collagen, a marker for hypertrophic 476 chondrocytes (Inada et al. 1999; Mitchell et al. 2013; Vijayakumar et al. 2013), but 477 failed to obtain any positive signal. On the other hand, although the total cell count 478 increased significantly from 4 to 12 months of age (Figure 5G), the percentage of 479 apoptotic cells also largely increased (Figure 6B) supporting the notion that zebrafish 480 lost more chondrocytes with aging. Furthermore, the 2-week BrdU labeling (Figure 481 6D) suggests that active chondrocyte proliferation is correlated with the growth and 482 homeostasis of hyaline cartilage. 
Previous studies suggest that cells at synovium, tendon, fat pad, and groove of

484 Ranvier might be the sites for origin of articular chondrocytes (Candela et al. 2014;

485 Karlsson et al. 2009; Ohlsson et al. 1992). Recent lines of evidence suggest that the

486 superficial cells in articular cartilage serve as stem cells to provide new chondrocytes

487 during the juvenile stages (Dowthwaite et al. 2004; Li et al. 2017; Taniguchi et al.

488 2009). However, there has not been solid evidence to suggest the existence of

489 chondrocytic stem cells in mature articular cartilage. Although zebrafish vertebral

490 cartilage was juxtaposited by a perichondrial-like structure (brackets in Figure 3C

491 and D) similar to superficial cells in the mammalian articular cartilage, we did not see

492 any evidence to suggest that these cells are stem cells, nor did we find any evidence

493 for other cells to participate in cartilage homeostasis. Interestingly, our BrdU pulse-

494 chase study showed that some labeling was retained in cells from 4-month-old

495 zebrafish (Figure 6E), but none of these cells were found in the vertebral column of

496 12-month-old zebrafish (data not shown). The current model suggests that BrdU

497 dilution via cell proliferation can sufficiently explain the loss of BrdU signal in the

498 chase experiment (Ganusov \& De Boer 2013; Tough \& Sprent 1994). Considering 499 that proliferative cells do exist in the vertebral cartilage, although at a very low level

500 (Figure 6D), these proliferative cells might go through multiple rounds of proliferation

501 once triggered. Accordingly, our attempt for immunohistochemistry using previously

502 reported stem cell marker for mammalian articular cartilage, HMGB2, also failed to

503 find any positively stained cells in the cartilage of 12-month-old zebrafish (data not

504 shown) (Taniguchi et al. 2009). Hence, it is possible that the homeostasis of mature

505 cartilage depends on the proliferation of terminally differentiated cells, but not stem

506 cells.

\section{CONCLUSIONS}

508 Taken together, the body maturity of zebrafish come much later than sexual 509 maturity. A simple exercise training system for zebrafish was designed and 510 demonstrated that short-term intensive swim exercise does not affect cartilage 511 homeostasis. However, similar to mammalian articular cartilage, the hyaline 512 cartilage of zebrafish exhibits different chondrocyte dynamics between young and 513 more mature stages. These results imply that aging perturbs chondrocyte 514 homeostasis and in turn lead to cartilage degeneration.

\section{ACKNOWLEDGMENTS}

The authors would like to thank Dr. Harry Mersmann for proofreading and 517 revising this manuscript, and Dr. Yun-Jin Jiang as well as Dr. Ching-Ho Wu for their 518 constructive discussions of this work. We would also like to acknowledge the 519 technical supports from Dr. Chih-Hsien Chiu on histological preparation, Mr. Ting520 Hao Wang and the Imaging Core Facility of Taipei Medical University on high521 throughput imaging and analysis, Dr. Wei-Cheng Chang, Mr. Hong-Wen Huang and 522 National Laboratory Animal Center on microCT imaging and analysis, Technology 
523 Commons, College of Life Science, National Taiwan University on the scanning

524 electron microscopy, and Ms. Ting-Yu Tseng on confocal microscopy.

\section{REFERENCES}

526

527

528

529

530

531

532

533

534

535

536

537

538

539

540

541

542

543

544

545

546

547

548

549

550

551

552

553

554

555

556

557

558

559

560

561

562

563

564

565

566

Alsalameh S, Amin R, Gemba T, and Lotz M. 2004. Identification of mesenchymal progenitor cells in normal and osteoarthritic human articular cartilage. Arthritis and Rheumatism 50:1522-1532. 10.1002/art.20269

Arokoski J, Kiviranta I, Jurvelin J, Tammi M, and Helminen HJ. 1993. Long-distance running causes site-dependent decrease of cartilage glycosaminoglycan content in the knee joints of beagle dogs. Arthritis and Rheumatism 36:1451-1459.

Barbero A, Grogan S, Schafer D, Heberer M, Mainil-Varlet P, and Martin I. 2004. Age related changes in human articular chondrocyte yield, proliferation and post-expansion chondrogenic capacity. Osteoarthritis and Cartilage 12:476-484. 10.1016/j.joca.2004.02.010

Bennell KL, Malcolm SA, Khan KM, Thomas SA, Reid SJ, Brukner PD, Ebeling PR, and Wark JD. 1997. Bone mass and bone turnover in power athletes, endurance athletes, and controls: a 12-month longitudinal study. Bone 20:477-484.

Bird NC, and Mabee PM. 2003. Developmental morphology of the axial skeleton of the zebrafish, Danio rerio (Ostariophysi: Cyprinidae). Developmental Dynamics 228:337357. $10.1002 /$ dvdy. 10387

Busija L, Bridgett L, Williams SR, Osborne RH, Buchbinder R, March L, and Fransen M. 2010. Osteoarthritis. Best Practice \& Research: Clinical Rheumatology 24:757-768. 10.1016/j.berh.2010.11.001

Candela ME, Cantley L, Yasuaha R, Iwamoto M, Pacifici M, and Enomoto-Iwamoto M. 2014. Distribution of slow-cycling cells in epiphyseal cartilage and requirement of betacatenin signaling for their maintenance in growth plate. Journal of Orthopaedic Research 32:661-668. 10.1002/jor.22583

Chang NY, Chan YJ, Ding ST, Lee YH, HuangFu WC, and Liu IH. 2016. Sterol OAcyltransferase 2 Contributes to the Yolk Cholesterol Trafficking during Zebrafish Embryogenesis. PloS One 11:e0167644. 10.1371/journal.pone.0167644

Davidson EA, and Small W. 1963. Metabolism in vivo of connective-tissue mucopolysaccharides. I. Chondroitin sulfate $\mathrm{C}$ and keratosulfate of nucleus pulposus. Biochimica et Biophysica Acta 69:445-452.

Dowthwaite GP, Bishop JC, Redman SN, Khan IM, Rooney P, Evans DJ, Haughton L, Bayram Z, Boyer S, Thomson B, Wolfe MS, and Archer CW. 2004. The surface of articular cartilage contains a progenitor cell population. Journal of Cell Science 117:889-897. 10.1242/jcs.00912

Ferry B, Lespessailles E, Rochcongar P, Duclos M, and Courteix D. 2013. Bone health during late adolescence: effects of an 8-month training program on bone geometry in female athletes. Joint, Bone, Spine: Revue du Rhumatisme 80:57-63. 10.1016/j.jbspin.2012.01.006

Fiaz AW, Leon-Kloosterziel KM, Gort G, Schulte-Merker S, van Leeuwen JL, and Kranenbarg S. 2012. Swim-training changes the spatio-temporal dynamics of skeletogenesis in zebrafish larvae (Danio rerio). PloS One 7:e34072. 10.1371/journal.pone.0034072 
567 Fontaine E, Lentink D, Kranenbarg S, Muller UK, van Leeuwen JL, Barr AH, and Burdick

568 JW. 2008. Automated visual tracking for studying the ontogeny of zebrafish

569 swimming. Journal of Experimental Biology 211:1305-1316. 10.1242/jeb.010272

570

571

572

Ganusov VV, and De Boer RJ. 2013. A mechanistic model for bromodeoxyuridine dilution naturally explains labelling data of self-renewing $\mathrm{T}$ cell populations. Journal of The Royal Society Interface 10:20120617. 10.1098/rsif.2012.0617

573

574

575

Gilbert MJ, Zerulla TC, and Tierney KB. 2014. Zebrafish (Danio rerio) as a model for the study of aging and exercise: physical ability and trainability decrease with age. Experimental Gerontology 50:106-113. 10.1016/j.exger.2013.11.013

576

577

578

579

580

581

582

583

584

585

586

587

588

589

590

591

592

593

594

595

596

597

598

599

600

601

602

603

604

605

606

607

608

609

610

611

612

613

614

Grogan SP, Miyaki S, Asahara H, D'Lima DD, and Lotz MK. 2009. Mesenchymal progenitor cell markers in human articular cartilage: normal distribution and changes in osteoarthritis. Arthritis Research \& Therapy 11:R85. 10.1186/ar2719

Hayes AJ, Reynolds S, Nowell MA, Meakin LB, Habicher J, Ledin J, Bashford A, Caterson B, and Hammond CL. 2013. Spinal deformity in aged zebrafish is accompanied by degenerative changes to their vertebrae that resemble osteoarthritis. PloS One 8:e75787. 10.1371/journal.pone.0075787

Hedlund H, Hedbom E, Heineg rd D, Mengarelli-Widholm S, Reinholt FP, and Svensson O. 1999. Association of the aggrecan keratan sulfate-rich region with collagen in bovine articular cartilage. Journal of Biological Chemistry 274:5777-5781.

Heinemeier KM, Schjerling P, Heinemeier J, Moller MB, Krogsgaard MR, Grum-Schwensen T, Petersen MM, and Kjaer M. 2016. Radiocarbon dating reveals minimal collagen turnover in both healthy and osteoarthritic human cartilage. Science Translational Medicine 8:346ra390. 10.1126/scitranslmed.aad8335

Huang WC, Hsieh YS, Chen IH, Wang $\mathrm{CH}$, Chang HW, Yang CC, Ku TH, Yeh SR, and Chuang YJ. 2010. Combined use of MS-222 (tricaine) and isoflurane extends anesthesia time and minimizes cardiac rhythm side effects in adult zebrafish. Zebrafish 7:297-304. 10.1089/zeb.2010.0653

Hur M, Gistelinck, C.A., Huber, P., Lee J., Thompson M.H. 2017. microCT-based skeletal phenomics in zebrafish reveals virtues of deep phenotyping at the whole-organism scale. bioRxiv.

Inada M, Yasui T, Nomura S, Miyake S, Deguchi K, Himeno M, Sato M, Yamagiwa H, Kimura T, Yasui N, Ochi T, Endo N, Kitamura Y, Kishimoto T, and Komori T. 1999. Maturational disturbance of chondrocytes in Cbfa1-deficient mice. Developmental Dynamics 214:279-290. 10.1002/(SICI)1097-0177(199904)214:4<279::AIDAJA1>3.0.CO;2-W

Jurvelin J, Helminen HJ, Lauritsalo S, Kiviranta I, Saamanen AM, Paukkonen K, and Tammi M. 1985. Influences of joint immobilization and running exercise on articular cartilage surfaces of young rabbits. A semiquantitative stereomicroscopic and scanning electron microscopic study. Acta Anatomica 122:62-68.

Karlsson C, Thornemo M, Henriksson HB, and Lindahl A. 2009. Identification of a stem cell niche in the zone of Ranvier within the knee joint. Journal of Anatomy 215:355-363. 10.1111/j.1469-7580.2009.01115.x

Kiviranta I, Tammi M, Jurvelin J, Saamanen AM, and Helminen HJ. 1988. Moderate running exercise augments glycosaminoglycans and thickness of articular cartilage in the knee joint of young beagle dogs. Journal of Orthopaedic Research 6:188-195. 10.1002/jor.1100060205

Kujala UM, Kaprio J, and Sarna S. 1994. Osteoarthritis of weight bearing joints of lower limbs in former elite male athletes. BMJ 308:231-234.

Peer] reviewing PDF | (2018:01:23253:3:0:CHECK 20 Aug 2018) 
615 Langenskiold A, Michelsson JE, and Videman T. 1979. Osteoarthritis of the knee in the

616

617

618

619

620

621

622

623

624

625

626

627

628

629

630

631

632

633

634

635

636

637

638

639

640

641

642

643

644

645

646

647

648

649

650

651

652

653

654

655

656

657

658

659

660

661

662

rabbit produced by immobilization. Attempts to achieve a reproducible model for studies on pathogenesis and therapy. Acta Orthopaedica Scandinavica 50:1-14.

Lee JY, Harvey WF, Price LL, Paulus JK, Dawson-Hughes B, and McAlindon TE. 2013. Relationship of bone mineral density to progression of knee osteoarthritis. Arthritis and Rheumatism 65:1541-1546. 10.1002/art.37926

Lequesne MG, Dang N, and Lane NE. 1997. Sport practice and osteoarthritis of the limbs. Osteoarthritis and Cartilage 5:75-86.

Li L, Newton PT, Bouderlique T, Sejnohova M, Zikmund T, Kozhemyakina E, Xie M, Krivanek J, Kaiser J, Qian H, Dyachuk V, Lassar AB, Warman ML, Barenius B, Adameyko I, and Chagin AS. 2017. Superficial cells are self-renewing chondrocyte progenitors, which form the articular cartilage in juvenile mice. FASEB Journal 31:1067-1084. 10.1096/fj.201600918R

Libby WF, Berger R, Mead JF, Alexander GV, and Ross JF. 1964. Replacement Rates for Human Tissue from Atmospheric Radiocarbon. Science 146:1170-1172.

Liu MJ, and Wang ZJ. 2013. [Adaptive changes of Zebrafish (Danio rerio) to anaerobic exercise training]. Dongwuxue Yanjiu 34:190-195.

Magkos F, Kavouras SA, Yannakoulia M, Karipidou M, Sidossi S, and Sidossis LS. 2007. The bone response to non-weight-bearing exercise is sport-, site-, and sex-specific. Clinical Journal of Sport Medicine 17:123-128. 10.1097/JSM.0b013e318032129d

Maimoun L, Coste O, Mura T, Philibert P, Galtier F, Mariano-Goulart D, Paris F, and Sultan C. 2013. Specific bone mass acquisition in elite female athletes. Journal of Clinical Endocrinology and Metabolism 98:2844-2853. 10.1210/jc.2013-1070

Mankin HJ, and Lippiello L. 1969. The turnover of adult rabbit articular cartilage. Journal of Bone and Joint Surgery (American Volume) 51:1591-1600.

McClelland GB, Craig PM, Dhekney K, and Dipardo S. 2006. Temperature- and exerciseinduced gene expression and metabolic enzyme changes in skeletal muscle of adult zebrafish (Danio rerio). Journal of Physiology 577:739-751. 10.1113/jphysiol.2006.119032

Mitchell RE, Huitema LF, Skinner RE, Brunt LH, Severn C, Schulte-Merker S, and Hammond CL. 2013. New tools for studying osteoarthritis genetics in zebrafish. Osteoarthritis and Cartilage 21:269-278. 10.1016/j.joca.2012.11.004

Muller UK, Stamhuis EJ, and Videler JJ. 2000. Hydrodynamics of unsteady fish swimming and the effects of body size: comparing the flow fields of fish larvae and adults. Journal of Experimental Biology 203:193-206.

Ohlsson C, Nilsson A, Isaksson O, and Lindahl A. 1992. Growth hormone induces multiplication of the slowly cycling germinal cells of the rat tibial growth plate. Proceedings of the National Academy of Sciences of the United States of America 89:9826-9830.

Parichy DM, Elizondo MR, Mills MG, Gordon TN, and Engeszer RE. 2009. Normal table of postembryonic zebrafish development: staging by externally visible anatomy of the living fish. Developmental Dynamics 238:2975-3015. 10.1002/dvdy.22113

Paukkonen K, Selkainaho K, Jurvelin J, Kiviranta I, and Helminen HJ. 1985. Cells and nuclei of articular cartilage chondrocytes in young rabbits enlarged after non-strenuous physical exercise. Journal of Anatomy 142:13-20.

Pritzker KP, Gay S, Jimenez SA, Ostergaard K, Pelletier JP, Revell PA, Salter D, and van den Berg WB. 2006. Osteoarthritis cartilage histopathology: grading and staging. Osteoarthritis and Cartilage 14:13-29. 10.1016/j.joca.2005.07.014

Peer] reviewing PDF | (2018:01:23253:3:0:CHECK 20 Aug 2018) 
663 Reimer MM, Sorensen I, Kuscha V, Frank RE, Liu C, Becker CG, and Becker T. 2008. Motor

664 neuron regeneration in adult zebrafish. Journal of Neuroscience 28:8510-8516.

$665 \quad$ 10.1523/JNEUROSCI.1189-08.2008

666 Rowlerson A, Radaelli G, Mascarello F, and Veggetti A. 1997. Regeneration of skeletal

667

668

669

670

671

672

673

674

675

676

677 muscle in two teleost fish: Sparus aurata and Brachydanio rerio. Cell and Tissue Research 289:311-322.

Saamamen AM, Kiviranta I, Jurvelin J, Helminen HJ, and Tammi M. 1994. Proteoglycan and collagen alterations in canine knee articular cartilage following $20 \mathrm{~km}$ daily running exercise for 15 weeks. Connective Tissue Research 30:191-201.

Saamanen AM, Tammi M, Jurvelin J, Kiviranta I, and Helminen HJ. 1990. Proteoglycan alterations following immobilization and remobilization in the articular cartilage of young canine knee (stifle) joint. Journal of Orthopaedic Research 8:863-873. 10.1002/jor.1100080612

Schmid TM, and Linsenmayer TF. 1985. Immunohistochemical localization of short chain cartilage collagen (type X) in avian tissues. Journal of Cell Biology 100:598-605.

678

679

Schneider CA, Rasband WS, and Eliceiri KW. 2012. NIH Image to ImageJ: 25 years of image analysis. Nature Methods 9:671-675.

680

681

Schumacher BL, Block JA, Schmid TM, Aydelotte MB, and Kuettner KE. 1994. A novel proteoglycan synthesized and secreted by chondrocytes of the superficial zone of articular cartilage. Archives of Biochemistry and Biophysics 311:144-152. 10.1006/abbi.1994.1219

683

684

685

686

687

688

689

690

691

692

693

694

695

696

697

698

699

700

701

702

703

704

705

706

707

708

Shimegi S, Yanagita M, Okano H, Yamada M, Fukui H, Fukumura Y, Ibuki Y, and Kojima I. 1994. Physical exercise increases bone mineral density in postmenopausal women. Endocrine Journal 41:49-56.

Siccardi AJ, 3rd, Padgett-Vasquez S, Garris HW, Nagy TR, D'Abramo LR, and Watts SA. 2010. Dietary strontium increases bone mineral density in intact zebrafish (Danio rerio): a potential model system for bone research. Zebrafish 7:267-273. 10.1089/zeb.2010.0654

Stockwell RA. 1967. The cell density of human articular and costal cartilage. Journal of Anatomy 101:753-763.

Taniguchi N, Carames B, Ronfani L, Ulmer U, Komiya S, Bianchi ME, and Lotz M. 2009. Aging-related loss of the chromatin protein HMGB2 in articular cartilage is linked to reduced cellularity and osteoarthritis. Proceedings of the National Academy of Sciences of the United States of America 106:1181-1186. 10.1073/pnas.0806062106

Tough DF, and Sprent J. 1994. Turnover of naive- and memory-phenotype T cells. Journal of Experimental Medicine 179:1127-1135.

Tveit M, Rosengren BE, Nilsson JA, and Karlsson MK. 2012. Former male elite athletes have a higher prevalence of osteoarthritis and arthroplasty in the hip and knee than expected. American Journal of Sports Medicine 40:527-533. $10.1177 / 0363546511429278$

van der Rest M, and Mayne R. 1988. Type IX collagen proteoglycan from cartilage is covalently cross-linked to type II collagen. Journal of Biological Chemistry 263:16151618.

Videman T, Eronen I, and Friman C. 1981. Glycosaminoglycan metabolism in experimental osteoarthritis caused by immobilization. The effects of different periods of immobilization and follow-up. Acta Orthopaedica Scandinavica 52:11-21. 
709 Vijayakumar P, Laize V, Cardeira J, Trindade M, and Cancela ML. 2013. Development of an 710 in vitro cell system from zebrafish suitable to study bone cell differentiation and 711 extracellular matrix mineralization. Zebrafish 10:500-509. 10.1089/zeb.2012.0833

712 Warming L, Hassager C, and Christiansen C. 2002. Changes in bone mineral density with 713 age in men and women: a longitudinal study. Osteoporosis International 13:105-112. $10.1007 / \mathrm{s} 001980200001$

715 Westerfield M. 2000. The zebrafish book : a guide for the laboratory use of zebrafish (Danio 716 rerio). Eugene, OR: M. Westerfield.

717 Zhong M, Carney DH, Boyan BD, and Schwartz Z. 2011. 17beta-Estradiol regulates rat 718 growth plate chondrocyte apoptosis through a mitochondrial pathway not involving 719 nitric oxide or MAPKs. Endocrinology 152:82-92. 10.1210/en.2010-0509 


\section{Table 1 (on next page)}

The maximal speeds of exercising zebrafish 
1

2

3 Table 1. The maximal speeds of exercising zebrafish

\begin{tabular}{cc}
\hline Zebrafish & Flow speed $(\mathrm{cm} / \mathrm{s})$ \\
\hline no.4 & 23.9 \\
no. 5 & 23.2 \\
no.10 & 24.0 \\
no.13 & 25.1 \\
no.15 & 23.4 \\
no.16 & 22.2 \\
no.17 & 22.0 \\
\hline
\end{tabular}

4

5 


\section{Figure 1}

Both age and intensive exercise affect growth of adult zebrafish

(A) An aquatic powerhead was connected to a clear water pipe to enforce intensive exercise in adult zebrafish. (B) The schedule for zebrafish underwent exercise-training consist of an 8hour exercise training session (red) two feeding sessions (blue) during the 14-hour light period and a resting period during the 10-hour dark period (black). (C) The body length of 12month-old zebrafish $(n=12)$ grew significantly compared to the 4-month-old zebrafish $(n=$ 8; Mann-Whitney's test). Data are presented as mean \pm SEM. (D) After intensive exercise (Exercise) for 14 days, body length of 12-month-old zebrafish was significantly shorter, while the zebrafish in control group (Control) was not (Wilcoxon matched-pairs signed rank test). (E) The body weight of 12-month-old zebrafish $(n=12)$ grew significantly compared to the 4month-old zebrafish ( $n=8$; Mann-Whitney's test). Data are presented as mean \pm SEM. (F) The body weight continued to grow in 14 days in 12-month-old (Control), but intensive exercise (Exercise) hindered this growth (Wilcoxon matched-pairs signed rank test). n.s.: not significant $(\mathrm{P}<0.05)$; *: $\mathrm{P}<0.05 ; * *: \mathrm{P}<0.01 ; * * *: \mathrm{P}<0.001 ; * * * * \mathrm{P}<0.0001$ 
A

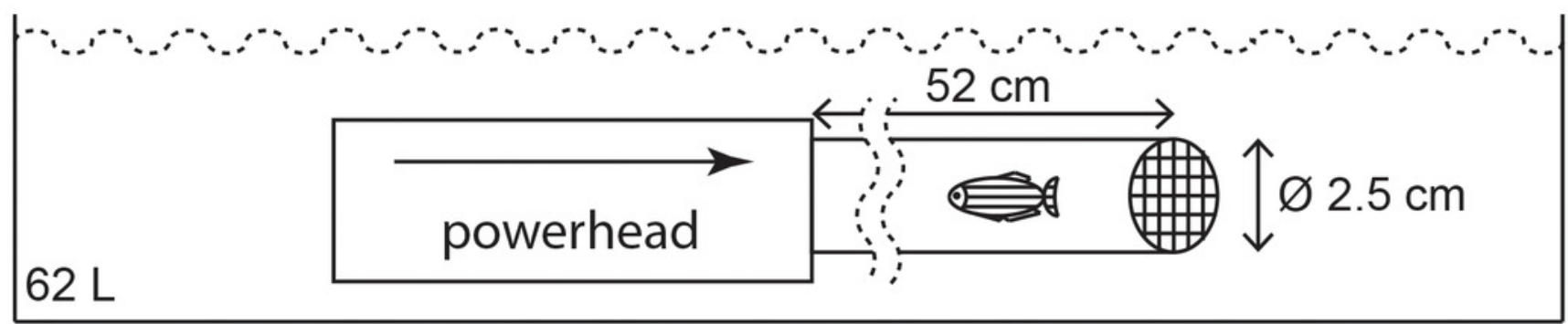

B

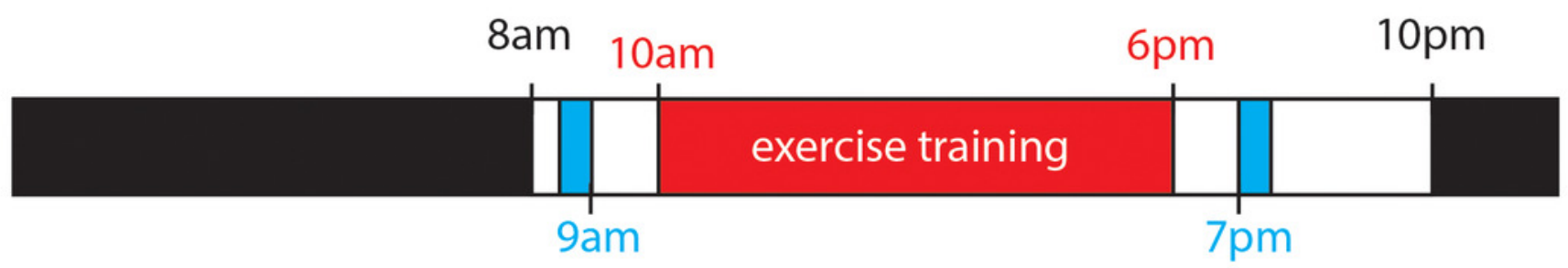

C

D
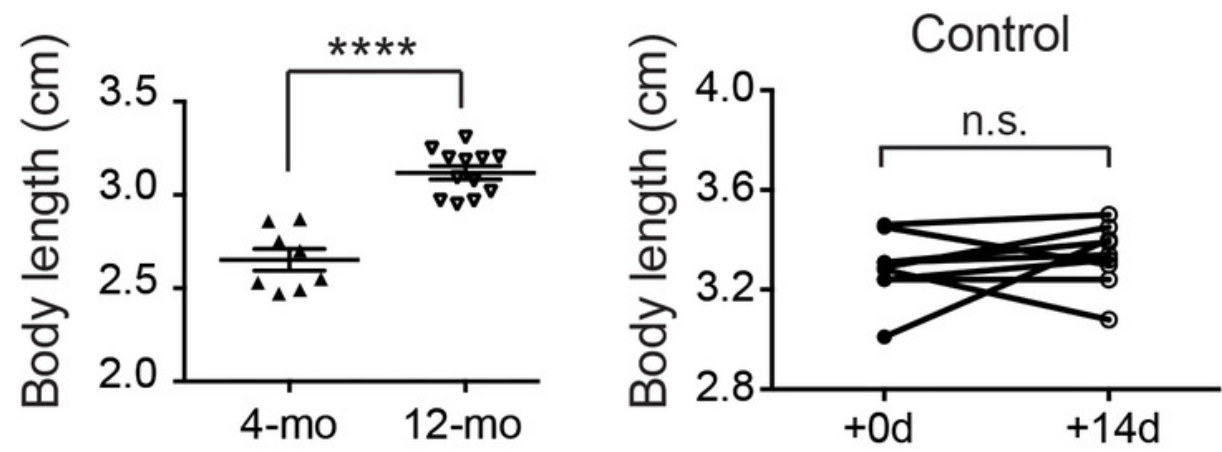

Exercise

E

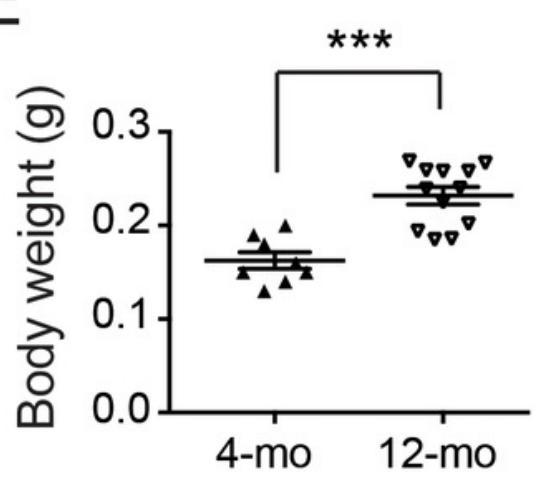

$\mathrm{F}$
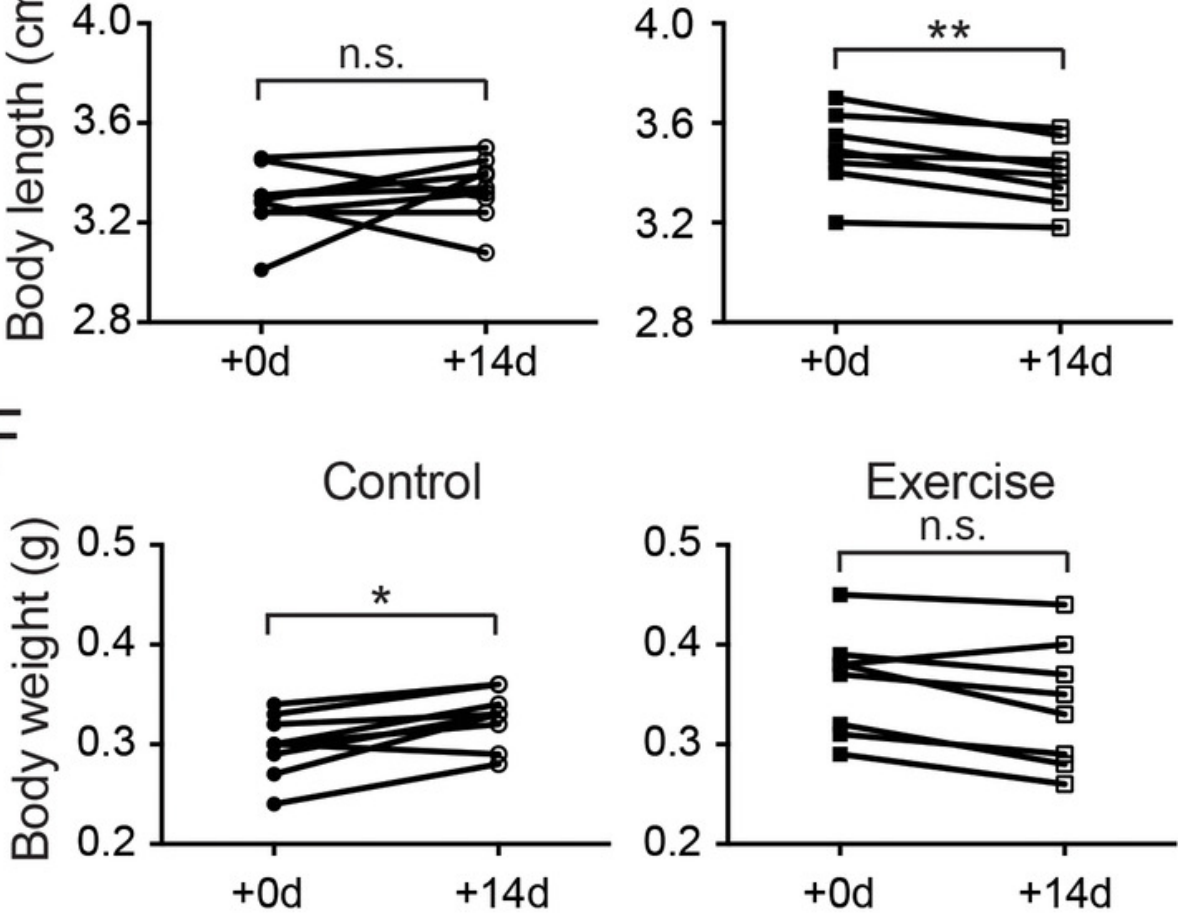


\section{Figure 2}

The accumulation of bone mineral density was affected by intensive exercise.
(A, B) Zebrafish were anesthetized for microCT to evaluate the BMD. A representative

transection microCT image of a zebrafish is shown (A). Note that only a cylindrical region (arrows in A) containing the hourglass-shaped fourth vertebrae was selected as region-ofinterest for quantitative analysis (B). The scale bars represent $1 \mathrm{~mm}(\mathrm{~A})$ and $0.15 \mathrm{~mm}$ (B). The color scale (0:dark purple; 255: white) represents -1000 to $3184 \mathrm{HU}$. (C, D) The BMD in the fourth vertebrae continued to increase in 12-month-old zebrafish during a 14-day period (C), but intensive exercise hindered this growth trend (D) (Wilcoxon matched-pairs signed rank test). n.s.: not significant ( $\mathrm{P}>0.05)$; **: $\mathrm{P}<0.01 ; * * * *$ : $<0.0001$
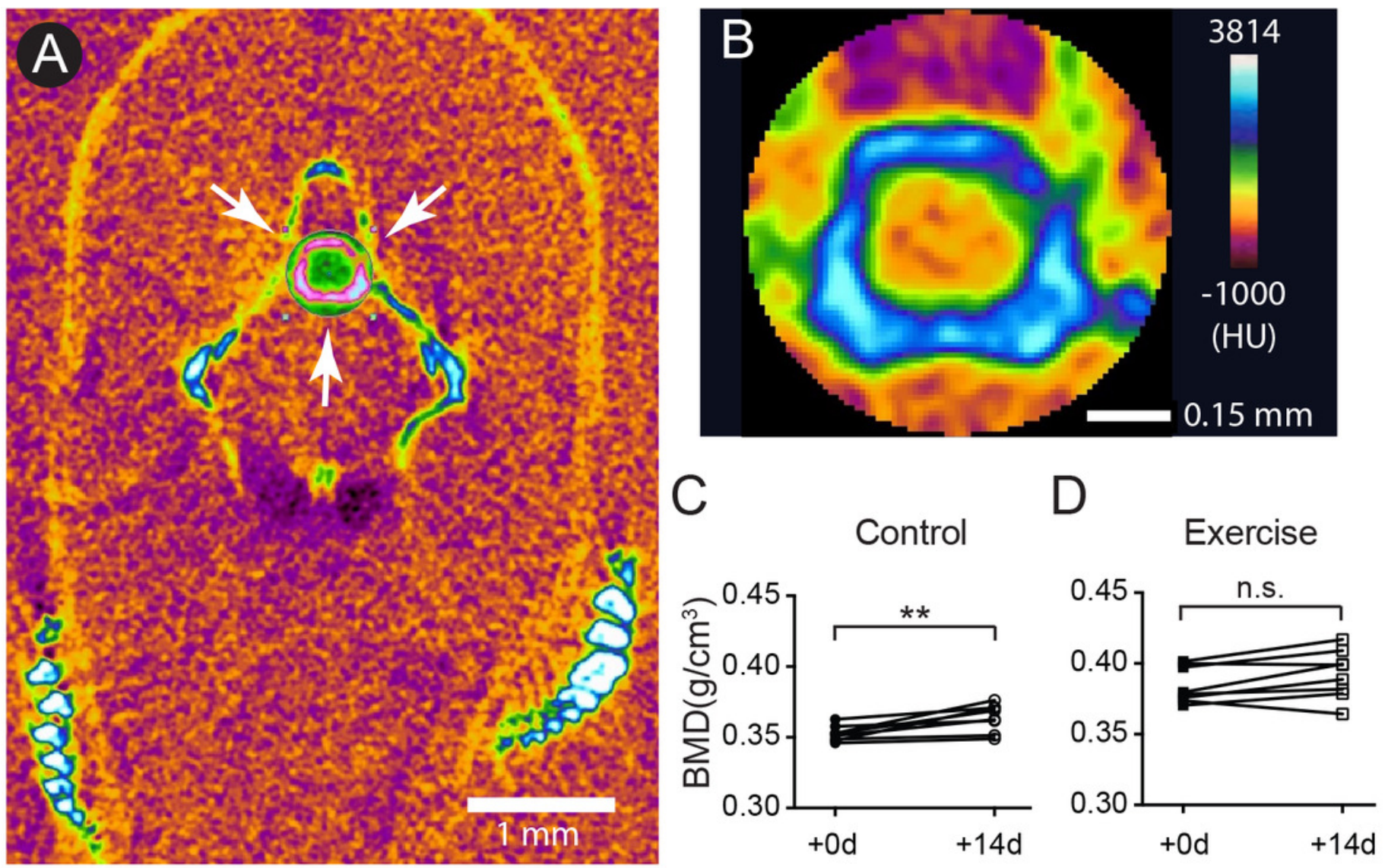


\section{Figure 3}

The fourth Weberian vertebra contained the largest hyaline cartilage among all the zebrafish vertebrae

Both immunohistochemistry against type II collagen (A) and histochemistry with safranin 0 and fast green (B) showed that the fourth Weberian vertebra (white arrows in A) contained the largest cartilage content in the spine. The scale bar represents $500 \mu \mathrm{m}$. (C) The H\&E histochemical staining showed typical hyaline cartilage features in the fourth Weberian vertebra. Note a cell-less fibrous region (bracket) is juxtaposited to the cell-rich region of the cartilage. The scale bar represents $50 \mu \mathrm{m}$. (D) A representative SEM image showed typical chondrocytes surrounded by lacunae covered by a fibrous perichondrial-like structure (arrow and bracket). The scale bar represents $10 \mu \mathrm{m}$. The yellow dotted box in (B) represents the approximate area shown in (C), while the yellow dotted box in (C) represents the approximate area shown in (D). 

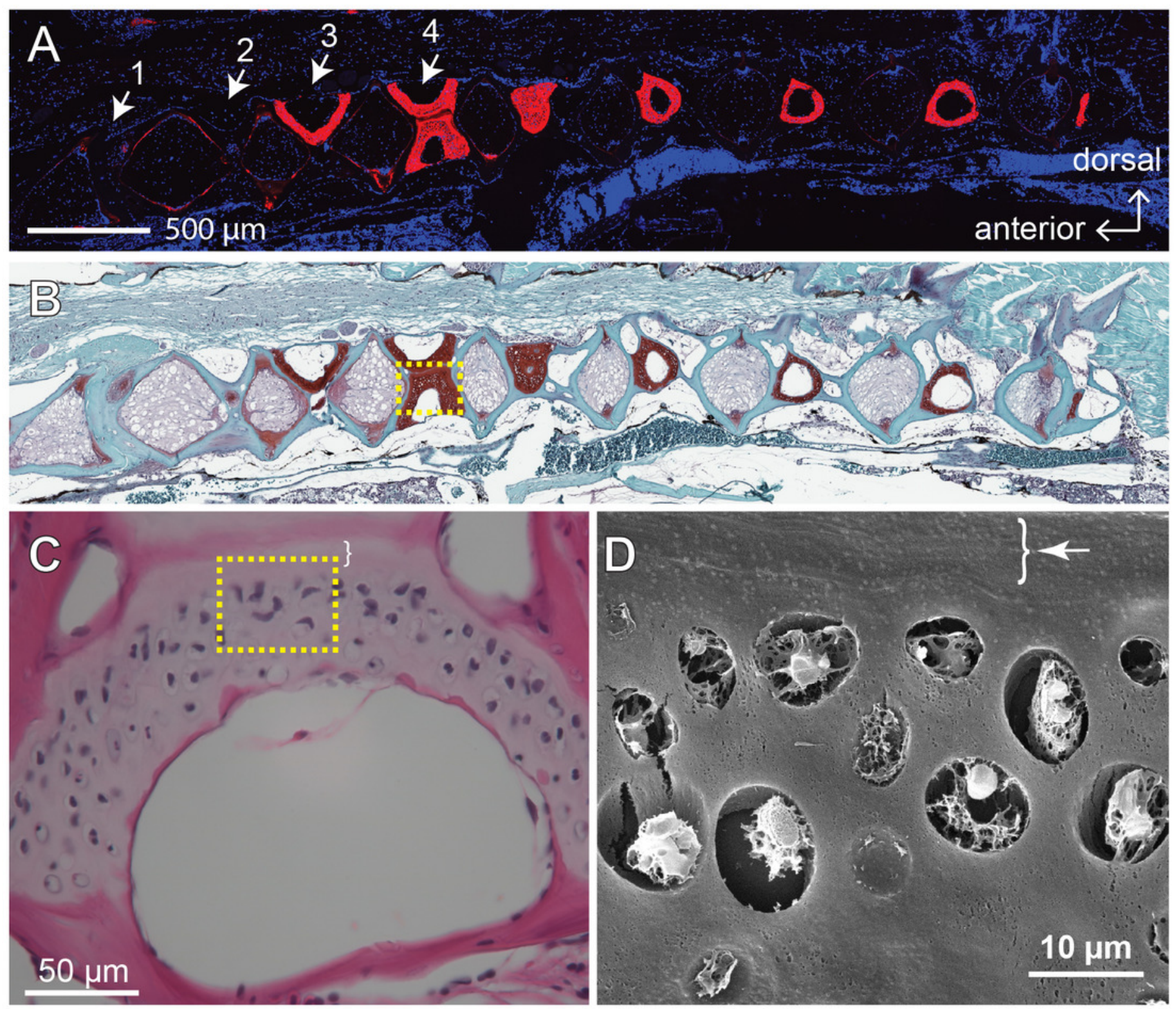


\section{Figure 4}

The type II collagen continues to accumulate in the spinal cartilage after sexual maturity

(A-C) The representative immunohistochemistry fluorescent micrographs showed distinct distributions of type II collagen in the spinal cartilage in 4-month-old (A) and 12-month-old (B, C) zebrafish. The scale bar represents $75 \mu \mathrm{m}$. (D-G) Three tissue slides across the sagittal sections of zebrafish vertebrae were obtained with a consistent interval between slides were selected from each subject for quantitative and statistical analysis. Both the occupying area (D) and average density (E) of type II collagen was significantly increased from $4(n=7)$ to 12 months of age ( $n=7$; Mann-Whitney test). However, the 14-day intensive exercise did not alter the content of type II collagen as both the area (F) and signal density (G) were comparable between the zebrafish in the control group $(n=6)$ and exercise group $(n=7)$. Data are presented as mean \pm SEM. n.s.: not significant $(\mathrm{P}>0.05)$; *: $\mathrm{P}<0.05$; ***: $\mathrm{P}<$ 0.001 


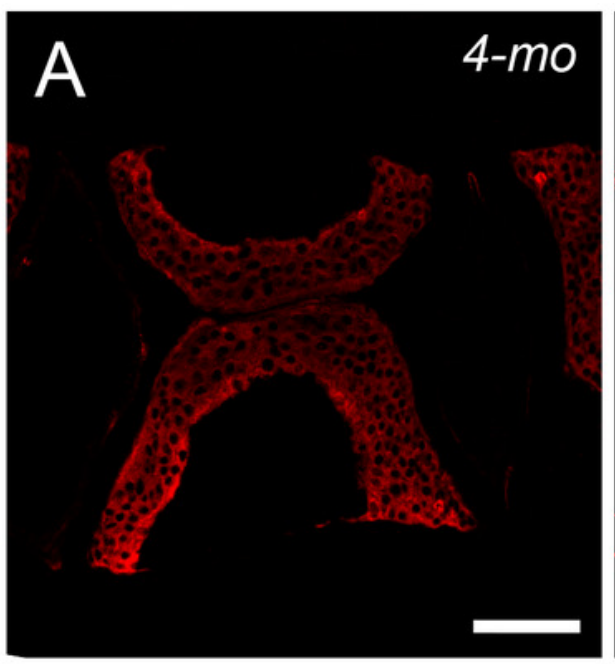

D

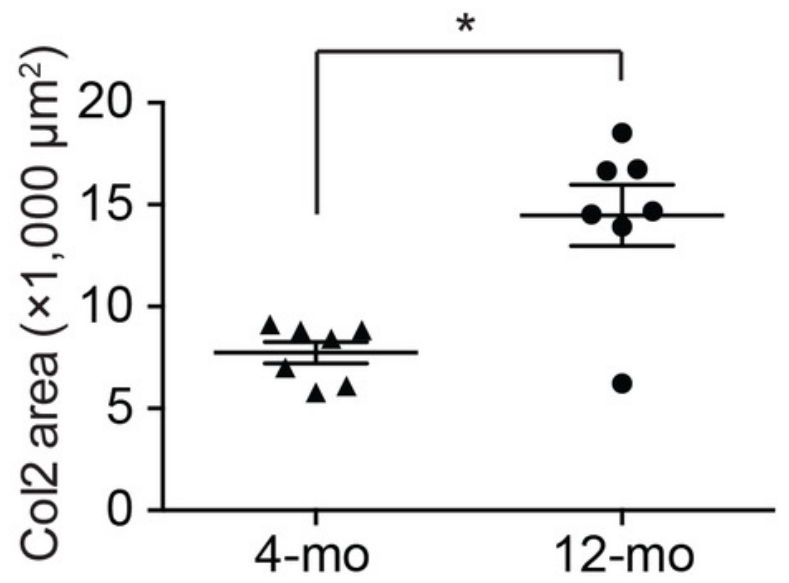

$\mathrm{F}$

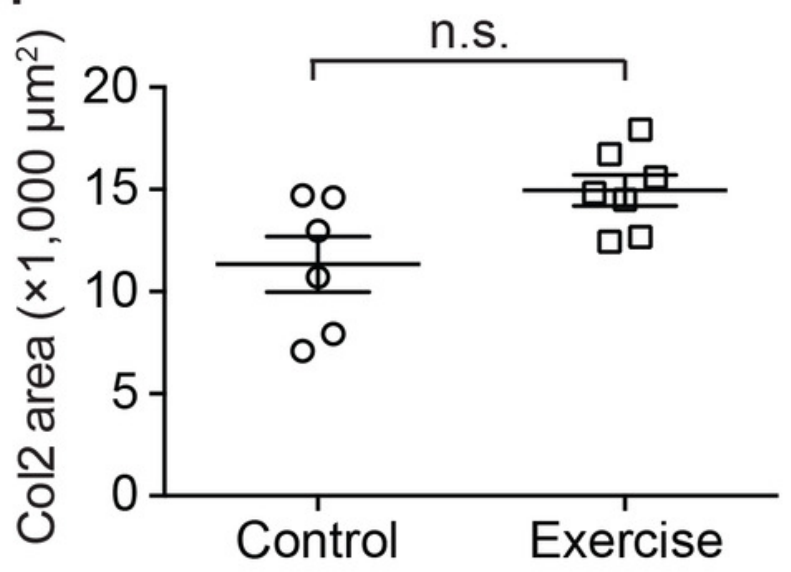

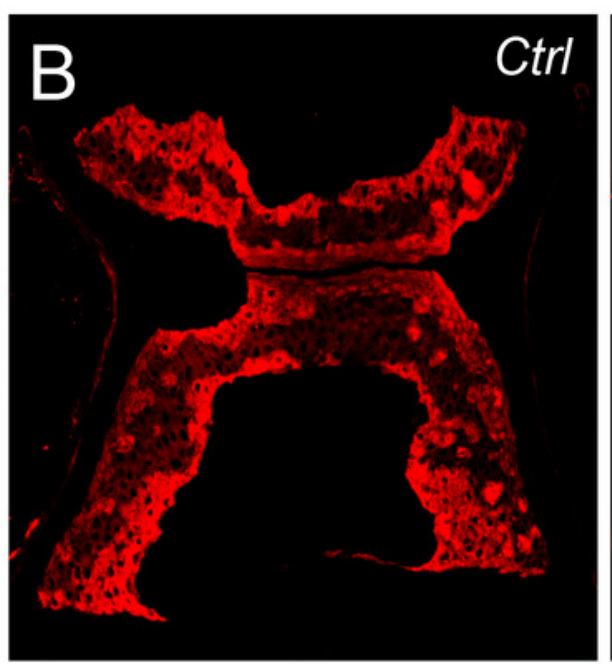

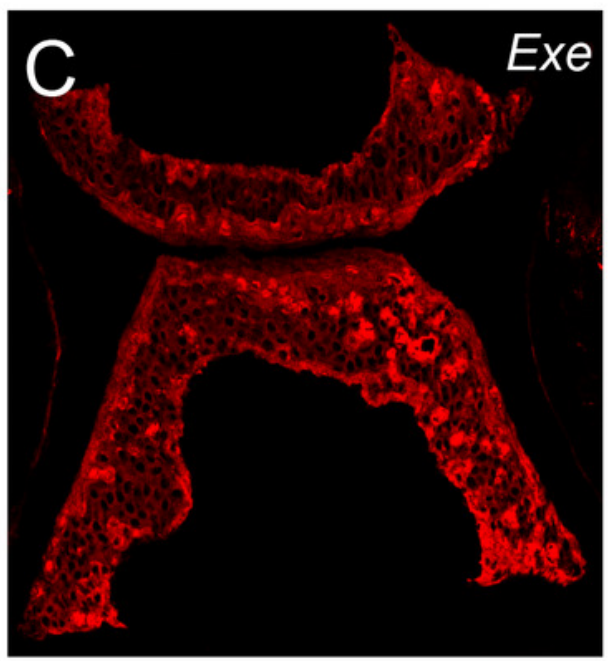

E

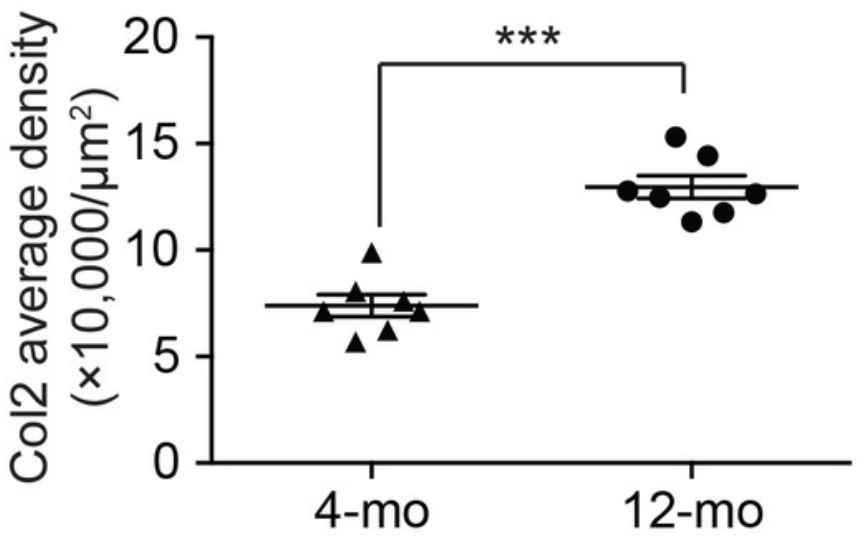

G

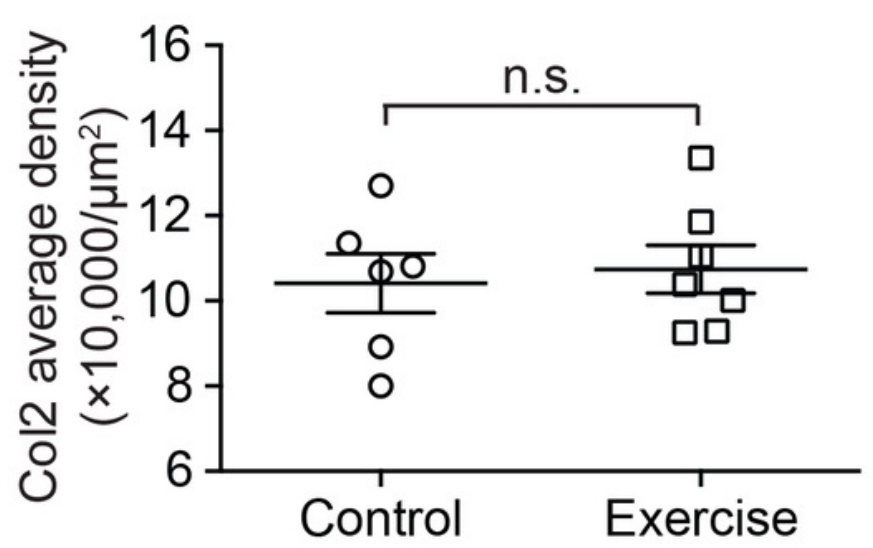




\section{Figure 5}

The cartilage ECM and chondrocytes varied as the zebrafish grew

(A-C) The representative histochemical micrographs showed the distribution patterns of

GAGs (red as stained by safranin O), collagen (cyan as stained by fast green) and cell nuclei (dark purple as stained by hematoxylin) in the spinal cartilage in 4-month-old $(A)$ and 12month-old (B, C) zebrafish. The yellow scale bar represents $100 \mu \mathrm{m}$. (D-G) Five tissue slides across the sagittal sections of zebrafish vertebrae obtained with a consistent interval between slides were selected from each subject for quantitative and statistical analysis. The occupying area of GAGs (D) was significantly increased from $4(n=8)$ to 12 months of age ( $n$ = 8; Mann-Whitney test) without affecting the averaged density ( $E$ ), but the 2-week intensive exercise-training $(n=8)$ did not alter the GAG content compared to the control group $(n=8)$ (Mann-Whitney test). The hematoxylin staining showed that the total chondrocyte number significantly increased from 4 to 12 months of age (G) (Mann-Whitney test) with a significantly decreased cellular density (F) (Mann-Whitney test). However, the 2-week intensive exercise-training did not affect chondrocyte distribution (Mann-Whitney test). Data are presented as mean \pm SEM. n.s.: not significant $(\mathrm{P}>0.05)$; *: $\mathrm{P}<0.05 ; * * *: \mathrm{P}<0.001$ 

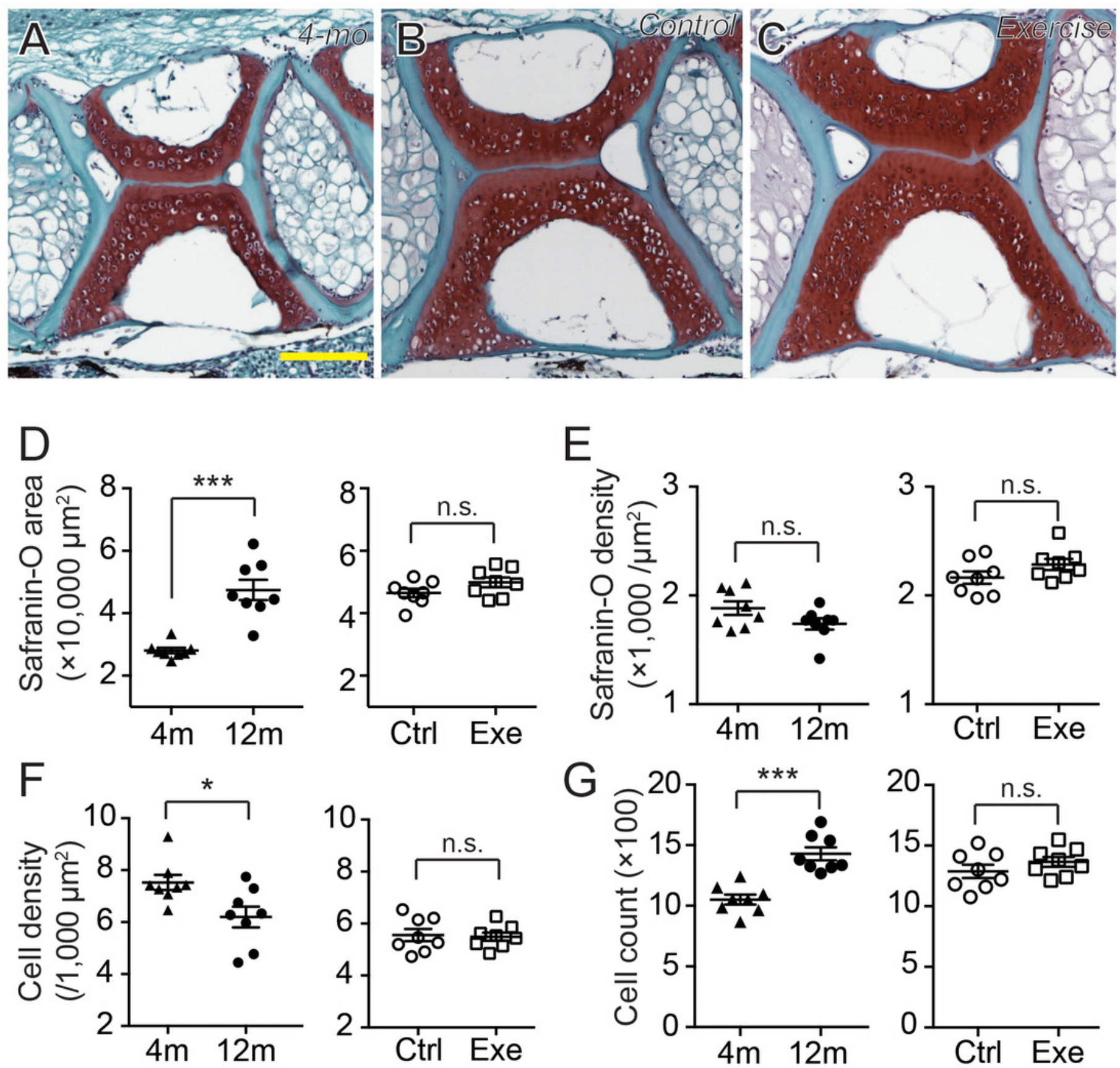


\section{Figure 6}

Chondrocytes dynamically turned over in the spinal cartilage

(A) A representative image of the differential interference contrast (DIC) and TUNEL fluorescent micrograph showed that the apoptotic cell nuclei (pink/white) could be distinguished from normal chondrocyte nuclei (blue as stained by DAPI). The scale bar represents $75 \mu \mathrm{m}$. (B) Quantitative analysis showed that the percentage of TUNEL positive nuclei in 12-month-old zebrafish ( $\mathrm{n}=8$ ) was significantly higher than in 4-month-old zebrafish ( $n=7$; Mann-Whitney test). However, the apoptotic cell rates were comparable in zebrafish with $(n=8)$ or without $(n=7)$ the 2-week exercise training. Data are presented as mean \pm SEM. (C) A representative image of the differential interference contrast (DIC) and immunohistochemistry against BrdU fluorescence. The proliferative cell nuclei (pink/white) could be distinguished from static chondrocyte nuclei (blue as stained by DAPI). (D) The percentage of BrdU positive nuclei in 4-month-old zebrafish $(n=8)$ was significantly higher than in 12-month-old zebrafish ( $n=11$; Mann-Whitney test). However, the apoptotic cell rates were comparable in zebrafish with $(n=8)$ or without $(n=8)$ the 2-week exercise training. Note that the axis scales are different in two different comparisons. Data are presented as mean \pm SEM. (E) After pulse-labeling BrdU for 15 days, the 4-month-old zebrafish was cleared from BrdU (chase) for 0, 15 and 30 days to locate the labeling retention cells. The presence of $\mathrm{BrdU}$ labeling retention cells tended to decrease with chase time, but the data were not significant (Kruskal-Wallis test). Chase periods were $0(n=8), 15$ $(n=6)$ and $30(n=6)$ days. $n . s .:$ not significant $(P>0.05) ; *: P<0.01 ; * * *: P<0.001$ 

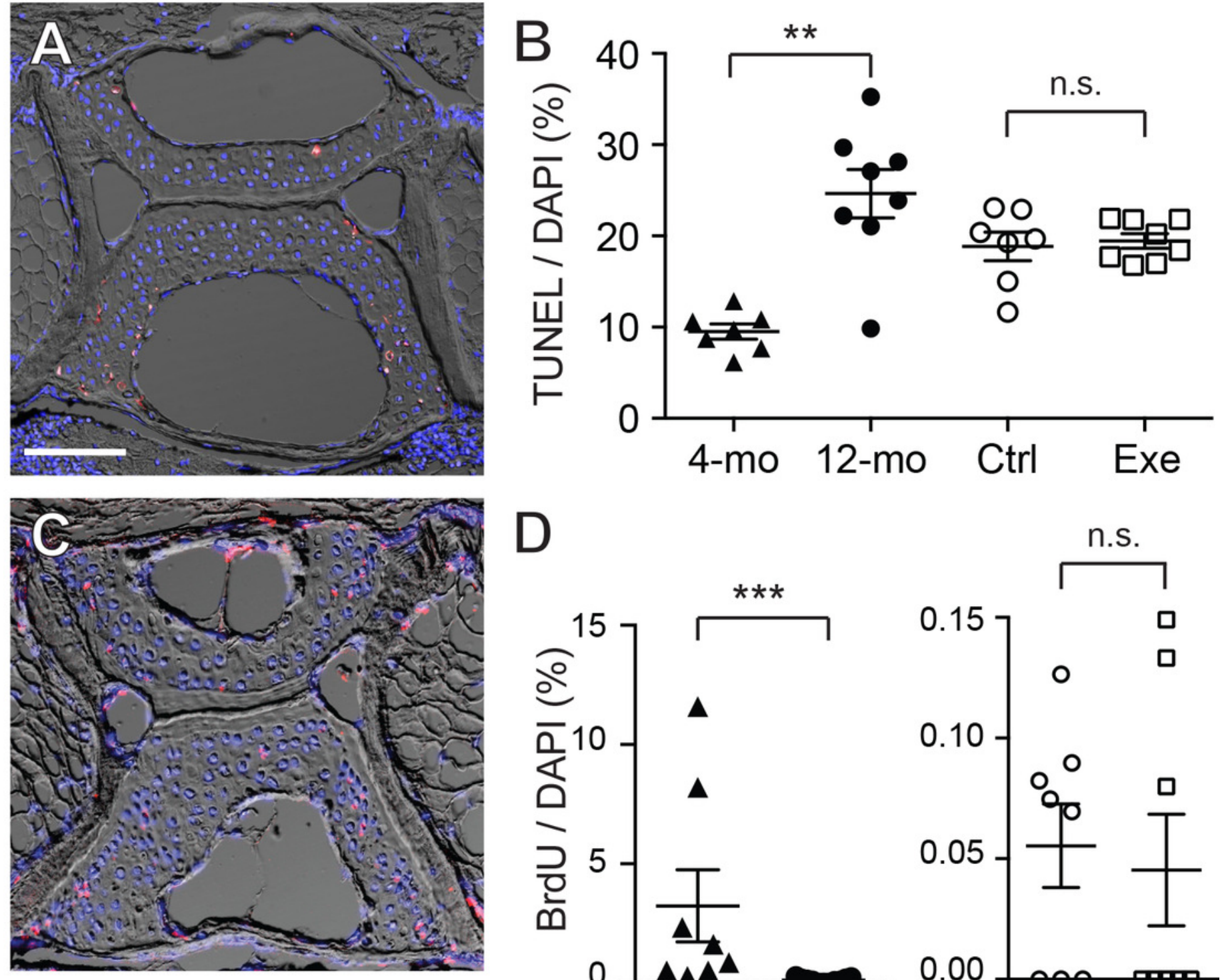

D
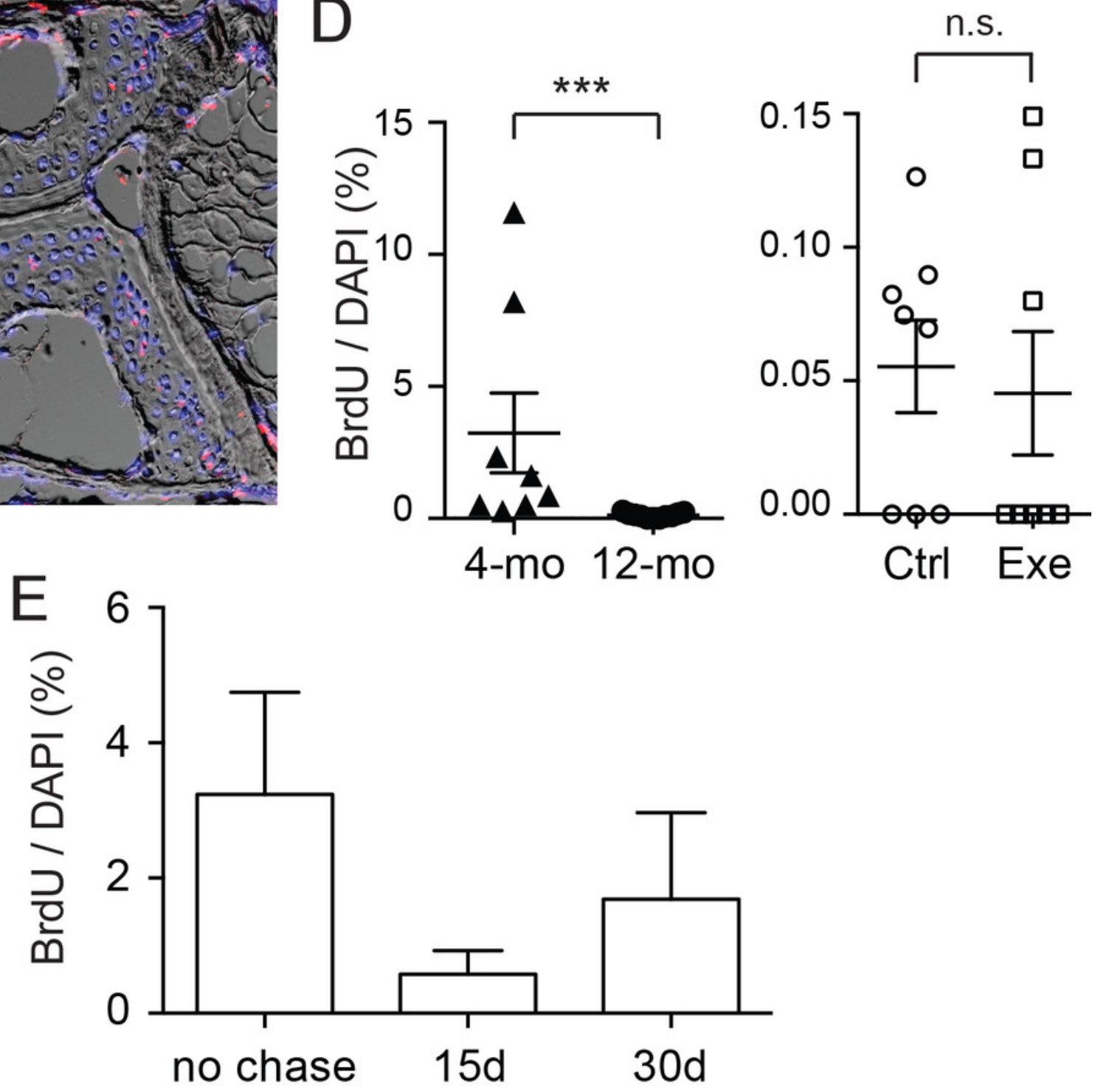